\title{
Next generation in vitro primary hepatic cell test systems - their suitability as an alternative to in vivo testing?
}

\author{
Ali Kermanizadeh ${ }^{1}$, Wolfgang Moritz $^{2}$ \\ ${ }^{1}$ Heriot Watt University, School of Engineering and Physical Sciences, Edinburgh, UK; ${ }^{2}$ InSphero, Schlieren, Switzerland \\ Correspondence to: Ali Kermanizadeh. Heriot Watt University, School of Engineering and Physical Sciences, Edinburgh, UK. \\ Email: Ali.Kermanizadeh@hw.ac.uk. \\ Comment on: Xiang C, Du Y, Meng G, et al. Long-term functional maintenance of primary human hepatocytes in vitro. Science 2019;364:399-402.
}

Submitted Aug 27, 2019. Accepted for publication Sep 09, 2019.

doi: 10.21037/hbsn.2019.09.09

View this article at: http://dx.doi.org/10.21037/hbsn.2019.09.09

To date traditional toxicity testing has relied heavily on inlife animal studies that are expensive and restrictive with regards to very important ethical implications. In addition, these studies often yield data that are not relevant to human exposures or biological responses due to intra-species variations, necessitating the requirement for further testing or candidate (drug) abandonment in late development stages. There is increasing global pressure from regulatory agencies, the general public, and the scientific community to develop in vitro alternatives for toxicity testing that can be utilized for mechanistic dose response hazard identification prior to embarking on small well designed and absolutely necessary in vivo studies.

The liver is often the first site of major drug metabolism after oral or intravenous administration and is a frontline target for drug-induced injury. Indeed, liver injury is a primary reason for drug regulation or withdrawal from the market. Common liver injuries due to xenobiotic exposure include cytotoxicity, proliferation, carcinogenesis, cholestasis, steatosis, fibrosis, and cirrhosis, all of which are typically assessed from in vivo histological analyses after long-term exposures. To recapitulate these complex phenotypes in vitro, organotypic test systems are required that represent relevant cell types in the liver, can maintain the physiologically necessary cell-cell signalling, and are viable for long time periods to allow these more complex pathological phenotypes to develop.

One of the conventional challenges with in vitro hepatic cell culture is the fact that human primary hepatocytes (PHH) have a relatively short half-life in 2D culture systems and often lose their liver-specific functions over time [cytochrome P450 (CYP450) activity, nitrogen excretion, albumin and urea production, etc.]. In recent years, considerable improvements in the in vitro longterm maintenance of liver function has been possible principally due the development of improved cell culture techniques principally by utilisation of $3 \mathrm{D}$ culture models such as collagen sandwich (1) and spheroids (2) and just as importantly the enhancement of optimal supplemented cell culture media which allow for long-term hepatic cell culture. These systems provide a microenvironment in which cell-cell and cell-matrix contact that mimic the physiological liver architecture.

To this end, a highly effective methodology for long-term culture of primary human hepatocytes in vitro was described by Xiang et al. (3). In the aforementioned study the authors demonstrate the culture of the cells in a 5 -factor supplemented (Forskolin, SB431542-inhibitor of ALK5, IWP2-WNT inhibitor, DAPT—notch inhibitor and LDN193189-ALK 2, 3 and 6 inhibitor) William E based culture medium which allowed for effective maintenance of metabolically and functionally active PHHs for a period of 4 weeks, primarily through inhibition of the epithelialmesenchymal transition (EMT) process commonly observed in PHH cultures. The authors show albumin secretion, urea synthesis and CYP450 activity in the PHHs cultured in the formulated medium over a period of 3 weeks, which was comparable to freshly isolated counterparts. Despite the impressive data presented in Xiang et al. it is important to state that there are now a number of different cell culture media formulations (some are commercially available) that allow for long-term culture of mono-culture of PHHs. 
Although hepatocytes have been the primary research focus for toxic responses in the liver, it is becoming increasingly clear that the non-parenchymal cells (NPCs) are essential in the liver in both homeostasis and disease and that they play a critical role in the in vivo response to xenobiotic insult $(4,5)$. Liver-resident Kupffer cell (KCs), for instance, are essential for homeostasis by constantly clearing systemic and gut-derived xenobiotics from the bloodstream and maintaining a tolerogenic environment. These macrophage cells are also important for sensing tissue injury, which drives their activation and subsequent release of various cytokines and chemokines that promotes an inflammatory milieu in the injured area of the liver. Activated KCs can further stimulate sinusoidal endothelial cells, and hepatic stellate cells to transition into myofibroblasts $(6,7)$. Depending on the extent of the damage, these cell-cell interactions can lead to hepatocyte death. NPCs are also fundamental in mitigating hepatic injury inflicted by xenobiotics by triggering repair processes up to complete liver regeneration after major trauma or surgical resections. Such repair processes largely rely on the TGF- $\beta$ axis which orchestrates the complex course of action during tissue remodelling. This holds also true for the progression of non-alcoholic fatty liver disease (NAFLD) into non-alcoholic steatohepatitis (NASH) and fibrosis. Both, chronic liver injury repair response and tissue remodelling during NASH progression is tightly associated with the pleiotropic activity of TGF- $\beta$ including epithelial to mesenchymal transition. Thus, permanent inhibition of the TGF- $\beta$ axis and associated EMT and the absence of modulating NPCs might compromise the ability of $\mathrm{PHH}$ to adequately respond to prolonged metabolic or xenobiotic stress.

As a side note, the addition of NPCs to in vitro hepatic test systems can also complicate the fine balance of required components in the cell culture medium not only to meet the nutritional and hormonal requirements of the different cell populations but also the biological effects these could have on the cells and the downstream consequences for toxicological and safety assessment of drugs/xenobiotics. As an example, the addition of Forskolin to hepatic cell culture medium significantly inhibits IL10 secretion from KCs in vitro.

In conclusion, the complex and crucial interplay between the various cell types in the liver cannot be replicated in a simplistic mono-culture in vitro models that are often utilized for chemical and drug-safety testing which highlights the necessity of the incorporation of NPC populations in vitro organotypic liver test systems. The 5 -factor supplementation of culture medium for the longterm preservation of hepatocyte function is a promising and effective approach for applications restricted to hepatocyte-only cultures. More physiological in vitro liver cultures including NPCs might depend on functional TGB- $\beta$ signalling and downstream processes in order to mimic chronic injury, diseased states and related repair mechanisms. Therefore, for the study of drug-induced chronic liver injury and related liver pathologies, the addition of chemical inhibitors affecting cellular plasticity needs to be considered with caution to preserve the repair and regenerative capacity of the liver.

\section{Acknowledgments}

None.

\section{Footnote}

Conflicts of Interest: The authors have no conflicts of interest to declare.

Ethical Statement: The authors are accountable for all aspects of the work in ensuring that questions related to the accuracy or integrity of any part of the work are appropriately investigated and resolved.

\section{References}

1. Roth AD, Lama P, Dunn S, et al. Polymer coating on a micropillar chip for robust attachment of PuraMatrix peptide hydrogel for 3D hepatic cell culture. Mater Sci Eng C Mater Biol Appl 2018;90:634-44.

2. Kermanizadeh A, Brown DM, Moritz W, et al. The importance of inter-individual Kupffer cell variability in the governance of hepatic toxicity in a $3 \mathrm{D}$ primary human liver microtissue model. Sci Rep 2019;9:7295.

3. Xiang C, Du Y, Meng G, et al. Long-term functional maintenance of primary human hepatocytes in vitro. Science 2019;364:399-402.

4. Krenkel O, Tacke F. Liver macrophages in tissue homeostasis and disease. Nat Rev Immunol 2017;17:306-21.

5. Grattagliano I, Bonfrate L, Diogo CV, et al. Biochemical mechanisms in drug-induced liver injury: certainties and 
doubts. World J Gastroenterol 2009;15:4865-76.

6. Preziosi ME, Monga SP. Update on mechanisms of liver regeneration. Semin Liver Dis 2017;37:141-51.

Cite this article as: Kermanizadeh A, Moritz W. Next generation in vitro primary hepatic cell test systems-their suitability as an alternative to in vivo testing? Hepatobiliary Surg Nutr 2020;9(1):103-105. doi: 10.21037/hbsn.2019.09.09
7. Woolbright BL, Jaeschke H. Mechanisms of inflammatory liver injury and drug induced hepatotoxicity. Curr Pharmacol Rep 2018;4:346-57. 